\title{
Führung auf Zeit
}

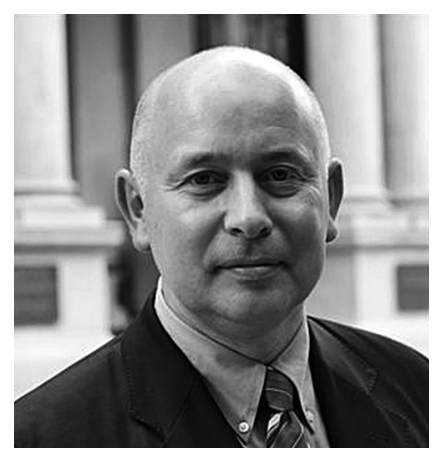

VON EDMUND FRÖHLICH Edmund Fröhlich absolvierte ein sozial- und erziehungswissenschaftliches Universitätsstudium, das er 1983 in München als Diplompädagoge abschloss. Danach war er im Management tätig, zunächst in der Freien Wohlfahrtspflege und seit 1991 in der Geschäftsführung von kommunalen und privaten Kliniken. Mit der Fröhlich Management $\mathrm{GmbH}$ ist er heute in der Geschäftsbesorgung für Unternehmen und Verbände der Sozial- und Gesundheitswirtschaft tätig.

www.froehlich-management.de

\author{
Das Interims-Management hat sich zunächst in akuten \\ Krisen einer Organisation oder eines Unternehmens \\ bewährt. Heute wird das Management auf Zeit auch \\ zur Überbrückung von personellen Ausfällen und \\ zeitweise als Bereichsleitung eingesetzt, um einen \\ Unternehmensteil zu sanieren. Gelegentlich werden \\ Interim-Manager auch im Rahmen von Projektarbeit \\ eingesetzt oder wenn es um eine Neuausrichtung \\ der Organisation oder des Unternehmens geht.
}

Die Nachricht vom kurzfristigen Ausscheiden des Heimleiters oder Geschäftsführers trifft den Nerv des gesamten Unternehmens. Gute Führungskräfte sind nicht so leicht zu finden, Auswahlverfahren brauchen ihre Zeit - Zeit, die kompetent überbrückt werden muss. Was in der Industrie seit langem üblich ist und auch in Akut- und Rehakliniken erfolgreich praktiziert wird, sehen inzwischen auch Sozialunternehmen als interessante Option: der Interimsmanager.

Ein alltägliches Beispiel: In vier Wochen soll der Erweiterungsbau im Rahmen eines Tages der offenen Tür der Öffentlichkeit vorgestellt werden. Bis dahin sind noch zahlreiche Entscheidungen zu treffen und organisatorische Probleme zu lösen. Ganz abgesehen vom offiziellen Teil der Veranstaltung mit Festrede. Pressekonferenz, Kontaktpflege mit Kassenvertretern und Geschäftspartnern. Und das alles ohne Führung? Ein Anflug von Panik erfasst nicht nur die Bereichsleiter, sondern auch die kommunalen Gewährsträger. Aber es muss nicht einmal eine derartige »Katastrophensituation« vorliegen, um an den kurzfristigen Einsatz eines externen Managers zu denken. Häufig reicht es schon, wenn eine Leitungsstelle regulär wieder nachbesetzt werden muss und eine Vakanz zu überbrücken ist.
Ein solcher »Interimsmanager « muss natürlich über langjährige Berufs- und Führungserfahrung verfügen. Er muss sicherstellen, dass die Betriebsabläufe reibungslos weiter funktionieren, weil ansonsten bereits nach wenigen Monaten Führungslosigkeit Strukturen entstehen, die vom neuen Leiter erst wieder mühsam korrigiert werden müssen und seine wertvolle Arbeitszeit binden. Führungslosigkeit ist teuer.

\section{Genaue Zieldefinition}

Noch ist das Interimsmanagement für soziale Einrichtungen wenig bekannt. Aber erste Erfahrungen zeigen, dass private und gemeinnützige Träger in schwierigen Umstrukturierungsphasen oder Krisensituationen klare Vorteile aus dem Einsatz eines Interimsmanagers ziehen: Seine Aufgaben und das Projekt werden exakt beschrieben. Der Zeitrahmen wird - meist zwischen drei und sechs Monaten - festgelegt und die Ziele werden vereinbart. Der Auftraggeber kauft darüber hinaus eine weitaus höhere Qualifikation ein, als sie ansonsten in der Einrichtung vorhanden ist.

Grundlage für diese Zusammenarbeit ist eine genaue Zieldefinition. Sobald diese festliegt, wird innerhalb der sozialen Einrichtung ein Projektteam zusammengestellt. Mit Hilfe des Interimsma- 
nagers werden dann systematisch Maßnahmen entwickelt und umgesetzt, die zur Zielerreichung führen. Es werden keine Lösungen vorgegeben, sondern die Teams müssen in die richtige Richtung motiviert werden. Hier wird sozusagen die Lücke geschlossen, die der Unternehmensberater nach seinem Abschlussbericht hinterlässt. Dabei geht es nicht um einseitige Personal- oder Sachkostenreduzierung, sondern um die Steigerung der Effizienz bei den wichtigsten Schnittstellen in den Bereichen Personal, Patienten, Dienstleistung, Prozesse und Öffentlichkeitsarbeit.

Es ist abzusehen, dass der Bedarf an Interimsmanagern in der Sozialwirtschaft weiter steigen wird. Folgende Beispiele sollen dies verdeutlichen:

Beispiel 1: Im Fall eines ganz normalen Stellenwechsels muss ein Nachfolger für den bisherigen Stelleninhaber gefunden werden. Gute Führungskräfte werden per Stellenanzeige gesucht oder von einem Personalberater direkt angesprochen. Neben der Zeit für den Auswahlprozess sind auch Kündigungsfristen zu beachten. Nachfolger können oft erst in drei bis sechs Monaten ihre neue Aufgabe übernehmen.

Beispiel 2: Eine größere private Unternehmensgruppe übernimmt im Rahmen ihrer Expansion ein neues Haus. Die Zeit der Suche nach einem passenden Geschäftsführer muss überbrückt werden.

Beispiel 3: Ein öffentlicher Träger muss sich aus Gründen, die nicht publik werden sollen, von seinem Direktor trennen. Der bisherige Stelleninhaber bleibt vorläufig im Amt und die Stelle soll nicht offiziell ausgeschrieben werden, weil die Absicht, sich zu trennen, nicht bekanntwerden soll und man auch niemanden brüskieren will. Bis zur Klärung der Situation kann der Interimsmanager die Führungsaufgaben ersatzweise oder begleitend übernehmen.

Beispiel 4: Ein Wohlfahrtsverband hat eine Unternehmensberatung mit einer Schwachstellenanalyse beauftragt, die auch Vorschläge für die Beseitigung der Schwachstellen beinhaltet. Der Auftraggeber erwartet auch dafür Unterstützung. Wenn die Unternehmensberatung keine Ressourcen frei hat für die mehrmonatige Leitungsaufgabe, kann sie sich - quasi als Subunternehmer einen Interimsmanager »einkaufen«.

Beispiel 5: Ein Pflegeheim befindet sich in wirtschaftlicher Schieflage.
Die Verantwortlichen sind sich sicher, dass eine neue Leitung die Lage wieder verbessern kann. In diesem Fall übernimmt der Interimsmanager zusätzlich Restrukturierungsaufgaben - eventuell gemeinsam mit einem Unternehmensberater oder Wirtschaftsprüfer.

Beispiel 6: Wenn eine Insolvenz eingetreten ist und der lnsolvenzverwalter mit den Gläubigern den Sozialbetrieb führt, wird für das operative Management bis zur Schließung, zur Übergabe oder zum Verkauf eine erfahrene Leitung benötigt.

Derartige kurzfristig auftauchende personelle Engpässe im Führungsbereich kann ein Manager auf Zeit mit genau umschriebenen Aufgaben überbrücken helfen. Objektiv und unvoreingenommen kann er Probleme lösen, die nur schwer von Personen angegangen werden können, die stark im sozialen Netzwerk des Unternehmens eingebunden sind.

Neu ist diese Idee nicht: Bereits in den letzten Jahren wurden Externe für unterschiedliche Projekte und Aufgaben beispielsweise in Krankenhäuser geholt. Es begann mit EDV-Beratern und ging weiter mit einer Outsourcing-Welle im Facility- und Catering-Bereich. Managementverträge und Umwandlungen der Rechtsform sowie letztlich Privatisierungen, Fusionen und Klinikverkäufe erforderten weitere Schritte in diese Richtung. Zudem engagieren sich immer mehr Klinikketten auf dem Pflegemarkt, schließen sich mittelständische gemeinnützige Träger zu Konzernstrukturen zusammen oder suchen private Einzelbetreiber aus Altersgründen einen Nachfolger.

Das Ende dieser Umstrukturierungen ist nicht in Sicht. Dabei wird gerade der Mangel an gut ausgebildeten und erfahrenen Managern die Situation für die Träger verschärfen. Immer häufiger werden deshalb Manager auf Zeit die anfallenden Spezialaufgaben lösen müssen. Fachleute mit Erfahrungen bei der Änderung der Rechtsform oder in der Leitung von Sanierungsfällen werden da genauso gefragt sein, wie gestandene Personalmanager, die Entlassungen durchführen sowie Sozialpläne erarbeiten und umsetzen können oder Marketing- und Vertriebsprofis.

\section{Notwendige Kompetenzen}

Letztlich sind Interimsmanager $»$ Mitarbeiter auf Zeit", die ein hohes Maß an Führungserfahrung mitbringen und auch schwierige und weniger angenehme Aufträge kompetent übernehmen können. Diese Männer und Frauen haben meist langjährige und einschlägige $\mathrm{Be}$ rufserfahrung, suchen aber keine Festanstellung, sondern sehen in den immer wieder neuen Aufgaben eine abwechslungsreiche Herausforderung.

Dabei zählt einerseits das fachliche Know-how des Interimsmanagers, andererseits werden gerade auch an seine sozialen Fähigkeiten hohe Anforderungen gestellt. Er muss in unterschiedliche Unternehmenskulturen eintauchen und sich darin bewegen können.

Neben Überzeugungskraft und starker Durchsetzungsfähigkeit sind auch soziale Kompetenzen wie Konfliktbereitschaft und Begeisterungsfähigkeit gefragt. Ein gesunder Blick für die zwischenmenschlichen Probleme, die schlimmstenfalls die erfolgreiche Umsetzung von Vorhaben zum Scheitern bringen können, ist ebenfalls unabdingbar. Und natürlich das Bestreben, sich immer wieder erfolgreich mit innovativen Lösungen den neuen Herausforderungen zu stellen. Aber auch die Mobilität und Kompetenz in Sachen Selbstorganisation muss stimmen.

Denn es gilt: Ein Interimsmanager muss immer seinen Koffer gepackt haben.
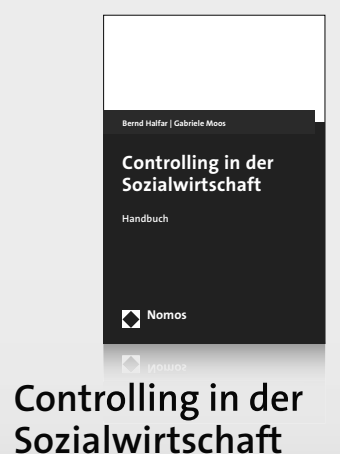

Handbuch

Von Bernd Halfar und Gabriele Moos 2013, ca. 300 S., brosch., ca. 34,- $€$ ISBN 978-3-8329-6327-9 Erscheint ca. Februar 2013 www.nomos-shop.de/13315

Nomos 